\title{
$\mathrm{ZnO}$ 纳米线/棒阵列的水热法制备及应用研究进展
}

\author{
郝锐 ${ }^{a, b}$ 邓霄*, $a, c$ 杨毅彪 ${ }^{a, c}$ 陈德勇 ${ }^{b}$ \\ $\left({ }^{a}\right.$ 太原理工大学物理与光电工程学院 太原 030024) \\ $\left({ }^{b}\right.$ 中国科学院电子学研究所 北京 100190$)$ \\ $\left({ }^{c}\right.$ 太原理工大学新型传感器与智能控制教育部与山西省重点实验室 太原 030024)
}

\begin{abstract}
摘要 氧化锌 $(\mathrm{ZnO})$ 纳米线/棒阵列的质量决定了所构建光电器件的性能. 为了制备出比表面积更大、垂直性更好以及 无根部融合的高质量 $\mathrm{ZnO}$ 纳米线/棒阵列, 本文概述了近几年两步水热法可控制备 $\mathrm{ZnO}$ 纳米线/棒阵列的研究进展, 分 别探讨了种子层、生长液和生长方法对纳米线/棒阵列形貌的影响, 详细分析了氨水、六次甲基四胺和聚乙烯亚胺对于 促进纳米线/棒阵列生长的作用机理, 提出了通过微流控技术可控制备 $\mathrm{ZnO}$ 纳米线阵列提高纳米线生长效率的方法. 最后介绍了 $\mathrm{ZnO}$ 纳米线/棒阵列的形貌对于提高染料敏化太阳能电池、纳米发电机、气体传感器和场发射器件性能的 重要作用, 并对未来两步水热法制备 $\mathrm{ZnO}$ 纳米线/棒阵列的发展趋势进行了展望.
\end{abstract}

关键词＼cjkstart氧化锌；纳米线/棒阵列; 两步水热法; 可控制备; 微流控技术

\section{Research Progress in Preparation and Applications of ZnO Nanowire/rod Arrays by Hydrothermal Method}

\author{
Hao, Rui ${ }^{a, b}$ Deng, Xiao ${ }^{*, a, c} \quad$ Yang, Yibiao $^{a, c} \quad$ Chen, Deyong $^{b}$ \\ ( ${ }^{a}$ College of Physics and Optoelectronics, Taiyuan University of Technology, Taiyuan 030024) \\ ( ${ }^{b}$ Institute of Electronics, Chinese Academy of Sciences, Beijing 100190) \\ ( ${ }^{c}$ Key Lab of Advanced Transducers and Intelligent Control System of the Ministry of Education and Shanxi Province, Tai- \\ yuan University of Technology, Taiyuan 030024)
}

\begin{abstract}
The quality of zinc oxide $(\mathrm{ZnO})$ nanowire/rod arrays determines the performance of the constructed photoelectric devices. In order to prepare high-quality $\mathrm{ZnO}$ nanowire/rod arrays with greater surface to volume ratio, better verticality and separate roots, a brief summary has been made about the research progress of the controllable preparation of $\mathrm{ZnO}$ nanowire/rod arrays using two-step hydrothermal method in this paper. Moreover, how the seed layer, growth solution and preparation method influence their morphology has been discussed respectively, and the mechanisms of ammonia, hexamethylenetetramine and polyethyleneimine in promoting the growth of nanowire/rod arrays have been particularly analyzed. At the same time, the method of controllable preparation of $\mathrm{ZnO}$ nanowire arrays based on microfluidic technique has been put forward, which can improve the growth efficiency of nanowire arrays. Finally, an introduction has been made about how the morphology of $\mathrm{ZnO}$ nanowire/rod arrays plays an important role in improving the performance of dye-sensitized solar cells, nanogenerators, gas sensors and field emission devices, and an outlook of future development trend of two-step hydrothermal method in preparing $\mathrm{ZnO}$ nanowire/rod arrays is also provided.
\end{abstract}

Keywords zinc oxide; nanowire/rod arrays; two-step hydrothermal method; controllable preparation; microfluidic technique

\section{1 引言}

氧化锌 $(\mathrm{ZnO})$ 是一种重要的 $\mathrm{II} \sim \mathrm{VI}$ 族直接带隙 $\mathrm{n}$ 型 半导体材料, 自然条件下具有纤锌矿的六方晶体结构, 晶格常数 $a=3.2496 \AA, c=5.2065 \AA$, 室温下的禁带宽度 为 $3.37 \mathrm{eV}$, 激子结合能为 $60 \mathrm{meV}$, 远高于 $\mathrm{ZnSe}$ 的 20 $\mathrm{meV}$ 和 $\mathrm{GaN}$ 的 $25 \mathrm{meV}^{[1 \sim 3]}$. 同时, $\mathrm{ZnO}$ 不仅具有优异的
光电和压电特性 ${ }^{[4]}$, 还具有极好的化学、机械和热稳定 性 ${ }^{[5]}$, 是一种具有巨大应用潜力的半导体材料.

$\mathrm{ZnO}$ 纳米线/棒阵列具有长径比高 ${ }^{\left[{ }^{[}\right]}$、比表面积大 ${ }^{[7]}$ 及电子注入效率高 ${ }^{[8]}$ 等优点, 在发光二极管 ${ }^{[9]}$ 、纳米发电 机 ${ }^{[10]} 、$ 染料敏化太阳能电池 ${ }^{[1]}$ 、紫外探测器 ${ }^{[12]}$ 和气体传 感器 ${ }^{[13]}$ 等领域具有重要应用. 高质量的 $\mathrm{ZnO}$ 纳米线/棒 阵列是提高器件性能的决定因素 ${ }^{[10,14]}$.

*E-mail: dengxiao@tyut.edu.cn; Tel.: 13834576609

Received August 20, 2014; published November 19, 2014

Project supported by the National Natural Science Foundation of China (Nos. 51205273, 61340053), the Scientific and Technological Innovation Programs of Higher Education Institutions in Shanxi (No. 20120007), the Youth Foundation of Taiyuan University of Technology (No. 2012L034) and the Excellent Innovation Programs for Postgraduate in Shanxi Province (No. 20133028).

项目受国家自然科学基金(Nos. 51205273, 61340053)、山西省高等学校科技创新基金(No. 20120007)、太原理工大学校青年基金(No. 2012L034)和山西 省研究生优秀创新基金(No. 20133028)资助. 
为了制备出高质量 $\mathrm{ZnO}$ 纳米线/棒阵列, 需要选择 良好的制备方法，而制备纳米线阵列的方法主要分为气 相法和液相法, 其中气相法包括碳热还原法 ${ }^{[15]}$ 、物理气 相沉积法 ${ }^{[16]}$ 和脉冲激光沉积法 ${ }^{[17]}$ 等, 而液相法包括水 热法 ${ }^{[18]}$ 、电化学沉积法 ${ }^{[19]}$ 和模板法 ${ }^{[20]}$ 等. 虽然气相法可 以制备出高质量的 $\mathrm{ZnO}$ 纳米线/棒阵列, 但该方法需要 高温、高真空、严格的操作程序和昂贵的仪器, 导致气 相法的生产成本很高, 限制了其在制备 $\mathrm{ZnO}$ 纳米线/棒 阵列方面的应用. 相比气相法而言, 水热法不仅可以大 规模生长出高质量的 $\mathrm{ZnO}$ 纳米线/棒阵列, 而且还具有 制备过程简单以及制备成本低等优点，使得水热法在制 备 $\mathrm{ZnO}$ 纳米线/棒阵列方面具有举足轻重的作用.

近年来基于两步水热法制备 $\mathrm{ZnO}$ 纳米线/棒阵列已 经得到了广泛而深入的研究, 本文在此基础上对近几年 两步水热法制备 $\mathrm{ZnO}$ 纳米线/棒阵列进行了总结, 归纳 了生长 $\mathrm{ZnO}$ 纳米线/棒阵列所采用的种子层制备方法、 生长液组分以及生长方法. 最后我们将微流控技术和传 统两步水热法相结合, 构建了微流控器件, 实现了 $\mathrm{ZnO}$ 纳米线阵列的快速制备, 解决了传统水热法不能快速制 备长度较长的 $\mathrm{ZnO}$ 纳米线阵列的问题.

\section{2 两步水热法可控制备 $\mathrm{ZnO}$ 纳米线/棒阵列}

水热法(hydrothermal method)属于液相法中的一种, 是指在密封的容器中以水作为溶剂, 并在一定温度和压 强条件下进行的一种化学反应 ${ }^{[21]}$. 自从 2003 年 Vayssieres 等 ${ }^{[22,23]}$ 首先将水热法应用于 $\mathrm{ZnO}$ 纳米棒阵列 的制备以来, 近几年基于水热法制备 $\mathrm{ZnO}$ 纳米线/棒阵 列的工艺得到了很大的发展和改进, 而目前基于两步水 热法在多种基底上可控制备高质量 $\mathrm{ZnO}$ 纳米线/棒阵列 受到了越来越多的关注. 两步水热法制备 $\mathrm{ZnO}$ 纳米线/ 棒阵列的关键是对包括种子层、生长液以及生长方法各 参数的有效调控 ${ }^{[14,15,24]}$, 控制好上述三个因素对于获得 高质量的 $\mathrm{ZnO}$ 纳米线/棒阵列至关重要.

\section{1 种子层}

两步水热法的第一步是在基底上制备一层 $\mathrm{ZnO}$ 薄 膜作为种子层，以减小纳米线/棒与基底之间的失配，从 而制备出排列更加整齐的 $\mathrm{ZnO}$ 纳米线/棒阵列 ${ }^{[25]}$. 而制 备 $\mathrm{ZnO}$ 种子层的方法有多种, 主要包括射频磁控浌射 法 ${ }^{[26]}$ 、脉冲激光沉积法 ${ }^{[27]}$ 、电沉积法 ${ }^{[8]}$ 以及溶胶一凝胶 法 ${ }^{[28]}$ 等. 通过优化种子层的制备方法以及种子液的配 比可以在后期生长出垂直均匀的 $\mathrm{ZnO}$ 纳米线/棒阵列.

2005 年, Greene 等 ${ }^{[15}$ 利用 $350{ }^{\circ} \mathrm{C}$ 热分解二水合醋 酸锌 $\left[\mathrm{Zn}\left(\mathrm{CH}_{3} \mathrm{COO}\right)_{2} \cdot 2 \mathrm{H}_{2} \mathrm{O}\right]$ 和乙醇 $\left(\mathrm{C}_{2} \mathrm{H}_{5} \mathrm{OH}\right)$ 混合溶液获 得了 $\mathrm{ZnO}$ 种子层, 并将覆盖此种子层的硅片作为基底, 然后利用六水合硝酸锌 $\left[\mathrm{Zn}\left(\mathrm{NO}_{3}\right)_{2} \cdot 6 \mathrm{H}_{2} \mathrm{O}\right]$ 和六次甲基四 胺 $\left[\left(\mathrm{CH}_{2}\right)_{6} \mathrm{~N}_{4}\right]$ 在 $90{ }^{\circ} \mathrm{C}$ 下制备出了垂直均匀的 $\mathrm{ZnO}$ 纳米 线阵列(图 1a), 并与浸涂预制的 $\mathrm{ZnO}$ 纳米晶体作为种子
层生长的纳米线阵列做了比较(图 1b), 结果表明基于热 分解醋酸锌的种子层所制备的纳米线阵列更加整齐, 而 且温度越高越有利于 $\mathrm{ZnO}$ 种子的结晶和生长, 过低的 热分解温度则会导致 $\mathrm{ZnO}$ 纳米线排列凌乱. 同时, 热分 解醋酸锌制备种子层所用基底材料可以为 $\mathrm{ZnO}$ 单晶、 透明导电氧化物(ITO 和 FTO)、无定形氧化物(玻璃和硅) 以及金属(金和钛)，可以在一定程度上降低实验的成本. 最后, 通过与乙酰丙酮锌和六水合硝酸锌种子液做比 较, 进一步表明了二水合醋酸锌种子液对生长垂直均匀 的 $\mathrm{ZnO}$ 纳米线阵列是必要的.

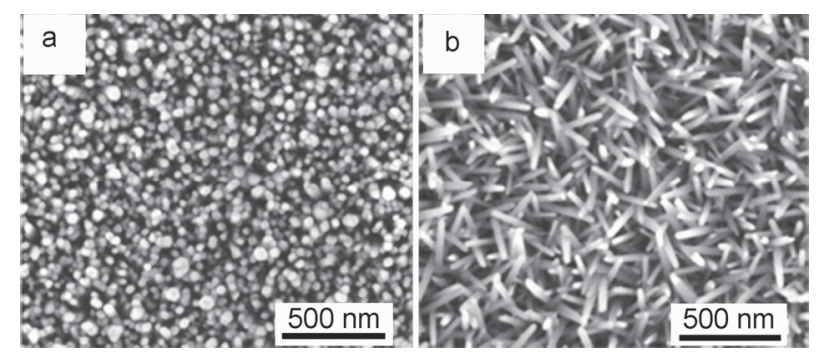

图 1 两步水热法制备的 $\mathrm{ZnO}$ 纳米线阵列的 SEM 俯视图 ${ }^{[15]}$

Figure 1 Top-view SEM images of $\mathrm{ZnO}$ nanowire arrays prepared by two-step hydrothermal method ${ }^{[15]}$

(a) $\mathrm{ZnO}$ seed layer from the thermal decomposition of zinc acetate dihydrate, (b) seed layer from the dip-coating of preformed $\mathrm{ZnO}$ nanocrystals

2011 年, Huang 等 ${ }^{[29]}$ 也利用二水合醋酸锌种子液在 $380{ }^{\circ} \mathrm{C}$ 热分解获得了种子层, 在 $12 \mathrm{~h}$ 内生长出的 $\mathrm{ZnO}$ 纳米棒阵列的长度达到了 $6 \mu \mathrm{m}$ 以上, 并探究了种子液 中的聚乙烯亚胺 $\left[\left(\mathrm{C}_{2} \mathrm{H}_{5} \mathrm{~N}\right)_{n}\right]$ 对 $\mathrm{ZnO}$ 纳米棒阵列的影响, 证明了不含聚乙烯亚胺的种子液能够长出排列更加整 齐的 $\mathrm{ZnO}$ 纳米棒阵列(图 2), 主要原因是种子液中的聚 乙烯亚胺会使种子层中晶核数量减少, 而基底上的种子 层又作为成核中心诱导纳米棒的生长, 最终在立体效 应 ${ }^{[30]}$ 的作用下使不含聚乙烯亚胺的种子液所制备出的 $\mathrm{ZnO}$ 纳米棒阵列排列更加紧密而抑制了倾斜.

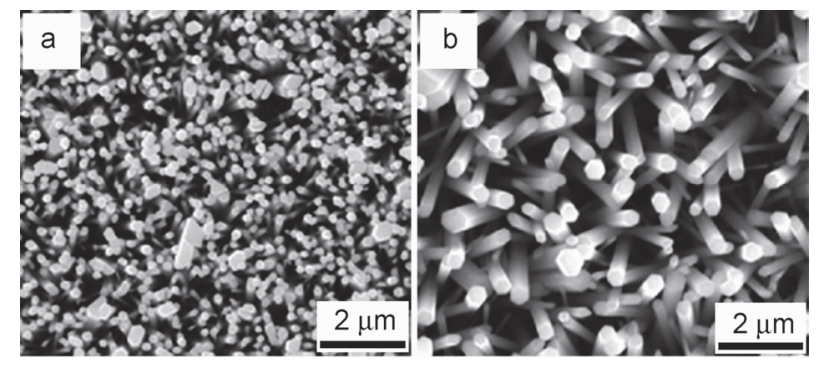

图 $2 \mathrm{ZnO}$ 纳米棒阵列的 $\mathrm{SEM}$ 图 ${ }^{[29]}$

Figure 2 SEM images of $\mathrm{ZnO}$ nanorod arrays ${ }^{[29]}$

(a) Seed solution without polyethyleneimine, (b) seed solution with polyethyleneimine

综上所述，可以通过热分解醋酸锌和乙醇的混合溶 液制备 $\mathrm{ZnO}$ 种子层, 而且所获得的种子层不仅适用于 水热法，也适用于其他生长技术制备垂直均匀的 $\mathrm{ZnO}$ 纳米线/棒阵列 ${ }^{[15]}$. 


\section{2 生长液}

两步水热法的第二步是利用生长液在覆盖 $\mathrm{ZnO}$ 种 子层的基底上生长 $\mathrm{ZnO}$ 纳米线/棒阵列, 生长液中的硝 酸锌 $\left[\mathrm{Zn}\left(\mathrm{NO}_{3}\right)_{2}\right]$ 可以为 $\mathrm{ZnO}$ 纳米线/棒的生长提供锌离 子, 而生长液中的其他溶质, 如氨水 $\left(\mathrm{NH}_{3} \cdot \mathrm{H}_{2} \mathrm{O}\right)$ 、六次甲 基四胺、氯化铵 $\left(\mathrm{NH}_{4} \mathrm{Cl}\right)$ 和聚乙烯亚胺, 对于 $\mathrm{ZnO}$ 纳米 线/棒阵列的生长质量也具有重要影响.

2009 年, $\mathrm{Yu}$ 等 ${ }^{[31]}$ 利用氨水-六水合硝酸锌作为生长 液, 在 $1 \mathrm{~h}$ 内制备出了平均直径约为 $500 \mathrm{~nm}$, 长度约为 7 $\mu \mathrm{m}$ 的 $\mathrm{ZnO}$ 纳米棒阵列(图 3). 实验表明, 氨水对 $\mathrm{ZnO}$ 纳 米棒阵列的生长起到了关键作用, 因为适当的氨水可以 抑制生长液中的均相成核, 减少溶质不必要的消耗. 这 一过程的反应机理为: 氨水不仅充当一种配位剂而产生 $\left[\mathrm{Zn}\left(\mathrm{NH}_{3}\right)_{4}\right]^{2+}$ (Eq. 1), 还可以充当一种弱碱(Eq. 2)而提 供 $\mathrm{ZnO}$ 纳米棒生长所需的碱性环境 ${ }^{[32]}$; 当对生长液加 热时, 溶液中的氨气会大量挥发而与溶液顶部的空气混 合(Eqs. 3, 4), 使溶液体系保持动态平衡. 通过控制反应 温度和对应的氨气压强, 可以抑制生长液中的均相成核 而促进 $\mathrm{ZnO}$ 种子层表面的异相成核, 从而制备出长度 较长的纳米棒阵列.

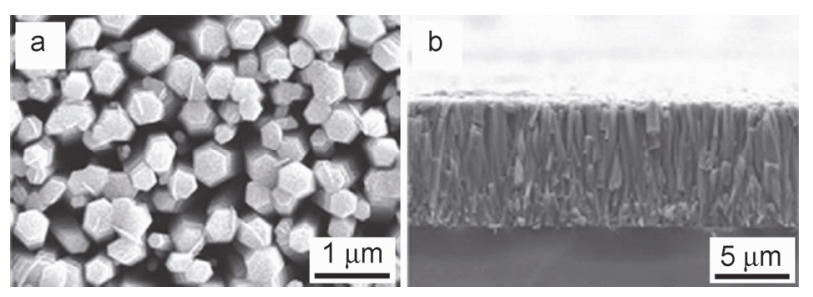

图 $3 \mathrm{ZnO}$ 纳米棒阵列的 SEM 图 ${ }^{[31]}$

Figure 3 SEM images of $\mathrm{ZnO}$ nanorod arrays ${ }^{[31]}$

(a) Top view, (b) side view

$$
\begin{aligned}
& \mathrm{Zn}^{2+}+4 \mathrm{NH}_{3} \leftrightarrow\left[\mathrm{Zn}\left(\mathrm{NH}_{3}\right)_{4}\right]^{2+} \\
& \mathrm{NH}_{3}+\mathrm{H}_{2} \mathrm{O} \leftrightarrow \mathrm{NH}_{4}^{+}+\mathrm{OH}^{-} \\
& {\left[\mathrm{Zn}\left(\mathrm{NH}_{3}\right)_{4}\right]^{2+} \rightarrow \mathrm{Zn}^{2+}+4 \mathrm{NH}_{3} \uparrow} \\
& \mathrm{Zn}^{2+}+2 \mathrm{OH}^{-} \rightarrow \mathrm{ZnO}+\mathrm{H}_{2} \mathrm{O}
\end{aligned}
$$

2006 年, Greene 等 ${ }^{[33]}$ 利用六次甲基四胺-硝酸锌作 为生长液制备出了长度约为 $2 \mu \mathrm{m}$, 平均长径比为 30 的 $\mathrm{ZnO}$ 纳米线阵列, 而生长时间仅为 $1.5 \mathrm{~h}$ (图 4a). 其生长 液中的六次甲基四胺充当一种 $\mathrm{pH}$ 缓冲剂而缓慢释放 $\mathrm{OH}^{-}$(Eqs. 5, 6), 生成的 $\mathrm{Zn}(\mathrm{OH})_{2}$ 在加热条件下分解为 $\mathrm{ZnO}$, 最终实现 $\mathrm{ZnO}$ 纳米线的生长 (Eqs. 7, 8) ${ }^{[34]}$. 同时, Sugunan 等 ${ }^{[22,35]}$ 发现生长液中的六次甲基四胺还可以充 当一种非极性面鳌合剂, 他会优先吸附于 $\mathrm{ZnO}$ 纳米线 的非极性面而抑制纳米线的径向生长，从而促进了
$\mathrm{ZnO}$ 纳米线沿[0001]晶向生长, 最终提高了 $\mathrm{ZnO}$ 纳米线 的长径比(图 4b).
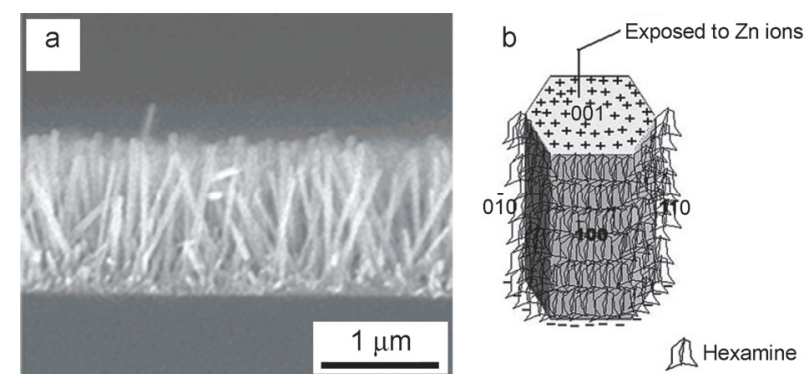

图 4 (a) $\mathrm{ZnO}$ 纳米线阵列的 SEM 侧面图 ${ }^{[33]}$ 及(b)六次甲基四胺吸附于 $\mathrm{ZnO}$ 纳米线的非极性表面的示意图 ${ }^{[35]}$

Figure 4 (a) Side-view SEM image of $\mathrm{ZnO}$ nanowire arrays ${ }^{[33]}$ and (b) schematic diagram of hexamethylenetetramine attaching to the non-polar facets of $\mathrm{ZnO}$ nanowire ${ }^{[35]}$

$$
\begin{aligned}
& \left(\mathrm{CH}_{2}\right)_{6} \mathrm{~N}_{4}+\mathrm{H}_{2} \mathrm{O} \stackrel{\Delta}{\longrightarrow} 4 \mathrm{NH}_{3} \uparrow+6 \mathrm{HCHO} \uparrow \\
& \mathrm{NH}_{3}+\mathrm{H}_{2} \mathrm{O} \rightarrow \mathrm{NH}_{4}^{+}+\mathrm{OH}^{-} \\
& \mathrm{Zn}^{2+}+2 \mathrm{OH}^{-} \leftrightarrow \mathrm{Zn}(\mathrm{OH})_{2} \downarrow \\
& \mathrm{Zn}(\mathrm{OH})_{2} \leftrightarrow \mathrm{ZnO} \downarrow+\mathrm{H}_{2} \mathrm{O}
\end{aligned}
$$

2014 年, Chen 等 ${ }^{[36]}$ 利用氨水一六次甲基四胺-六水 合硝酸锌作为生长液, 在 $8 \mathrm{~h}$ 内制备出了长度达到 20 $\mu \mathrm{m}$ 的 $\mathrm{ZnO}$ 纳米线阵列(图 5), 相应的生长液所占容器体 积比为 0.95 . 实验的关键在于通过控制容器中的溶液体 积来控制氨水的浓度，而适当增加氨水浓度可以促进 $\mathrm{ZnO}$ 纳米线的快速生长. $\mathrm{Gao}$ 等 $^{[8]}$ 也通过改变生长液中 的氨水含量证明了适当增加氨水浓度可以促进 $\mathrm{ZnO}$ 纳 米线的轴向生长, 使 $\mathrm{ZnO}$ 纳米线的长度由 $4.5 \mu \mathrm{m}$ 增加 到了 $14 \mu \mathrm{m}$, 长径比达到了 $100 \sim 120$.
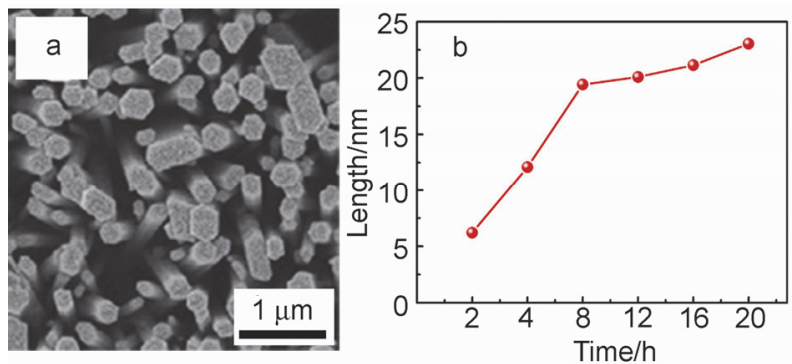

图 5 (a) $\mathrm{ZnO}$ 纳米线阵列的 $\mathrm{SEM}$ 俯视图及 (b) $\mathrm{ZnO}$ 纳米线阵列长度和 生长时间之间的函数关系 ${ }^{[36]}$

Figure 5 (a) Top-view SEM image of $\mathrm{ZnO}$ nanowire arrays and (b) the functional relationship between length of $\mathrm{ZnO}$ nanowire arrays and growth time ${ }^{[36]}$

2012 年, Cho 等 ${ }^{[10]}$ 利用氯化铵-六次甲基四胺-六水 合硝酸锌作为生长液, 在 $24 \mathrm{~h}$ 内制备出了长度为 13.6 $\mu \mathrm{m}$, 长径比达到 97.1 的 $\mathrm{ZnO}$ 纳米线阵列, 如图 6 所示. 分析认为, 生长液中的氯化铵作为一种缓冲剂, 其作用 
与氨水类似, 适当的氯化铵也可以降低反应溶液的过饱 和度而抑制均相成核, 减少溶质不必要的损耗, 从而促 进 $\mathrm{ZnO}$ 纳米线沿轴向生长.

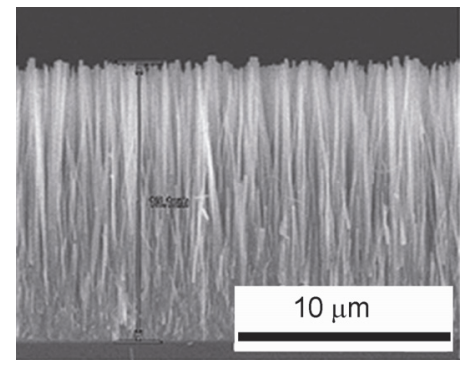

图 $6 \mathrm{ZnO}$ 纳米线阵列的 $\mathrm{SEM}$ 侧视图 ${ }^{[10]}$

Figure 6 Side-view SEM image of $\mathrm{ZnO}$ nanowire arrays ${ }^{[10]}$

2013 年, $\mathrm{Zhu}$ 等 ${ }^{[37]}$ 利用聚乙烯亚胺一六次甲基四胺硝酸锌作为生长液, 在 $32 \mathrm{~h}$ 内制备出了长度为 $11 \mu \mathrm{m}$ 的 $\mathrm{ZnO}$ 纳米线阵列, 当聚乙烯亚胺的浓度为 $7 \mathrm{mmol} \cdot \mathrm{L}^{-1}$ 时, 纳米线的长径比达到了 93(图 7a). 结果表明, 聚乙 烯亚胺对制备高长径比的 $\mathrm{ZnO}$ 纳米线具有至关重要的 作用, 因为聚乙烯亚胺是一种非极性高聚物, 具有大量 的侧面氨基 $\left(\mathrm{NH}_{2}\right)^{[38]}$, 在 $\mathrm{pH}$ 值为 $3 \sim 11$ 的范围内可以被 质子化而带上正电荷, 而初始的硝酸锌一六次甲基四胺 混合溶液的 $\mathrm{pH}$ 值为 6.2, 包含在聚乙烯亚胺被质子化的 $\mathrm{pH}$ 值范围内, 而且引入聚乙烯亚胺后溶液的 $\mathrm{pH}$ 值还会 随着其浓度的增加而成比例增加, 当生长液的 $\mathrm{pH}$ 值超 过 $\mathrm{ZnO}$ 的等电点 ${ }^{[32]}$ 时, $\mathrm{ZnO}$ 分解生成 $\left[\mathrm{Zn}(\mathrm{OH})_{4}\right]^{2-}$ 而使纳 米线的六个侧面带负电荷. 在静电力的作用下, 带正电 荷的聚乙烯亚胺会吸附到 $\mathrm{ZnO}$ 纳米线的侧面, 抑制纳 米线的径向生长, 从而提高纳米线的长径比(图 7b). 与 此同时, Zhou 等 ${ }^{[38,39]}$ 还证明了聚乙烯亚胺同样可以降低 生长液的过饱和度, 抑制生长液中的均相成核, 促进 $\mathrm{ZnO}$ 纳米线的轴向生长.

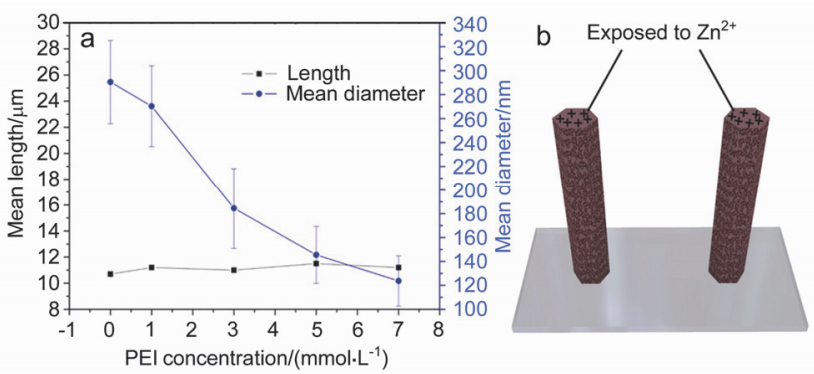

图 7 (a)不同浓度聚乙烯亚胺制备的 $\mathrm{ZnO}$ 纳米线阵列及(b)聚乙烯亚 胺吸附于 $\mathrm{ZnO}$ 纳米线表面的示意图 ${ }^{[37]}$

Figure 7 (a) $\mathrm{ZnO}$ nanowire arrays prepared with polyethyleneimine of different concentrations and (b) schematic diagram of polyethyleneimine attaching to the surfaces of $\mathrm{ZnO}$ nanowires ${ }^{[37]}$

2010 年, $\mathrm{Xu}$ 等 ${ }^{[14]}$ 利用氨水-聚乙烯亚胺-六次甲基 四胺一硝酸锌作为生长液, 在 $10 \mathrm{~h}$ 内制备出了长度超过 $30 \mu \mathrm{m}$ 的 $\mathrm{ZnO}$ 纳米线阵列, 但所制备的 $\mathrm{ZnO}$ 纳米线由于 太长而导致了纳米线根部的融合 (图 $8 \mathrm{a}$ ). 有文献指出, 造成这种现象的原因主要包括纳米线直径的扩大、纳米
线阵列的密度太高以及纳米线阵列根部所出现的短纳 米线 ${ }^{[24,40]}$. 虽然氨水可以降低生长液的过饱和度而减少 溶质不必要的消耗，但较高浓度的氨水也会抑制种子层 上的异相成核而阻碍 $\mathrm{ZnO}$ 纳米线的生长，所以可以通 过引入聚乙烯亚胺来抑制生长液中的均相成核，并维持 种子层上 $\mathrm{ZnO}$ 纳米线的高速生长, 主要原因归结为聚 乙烯亚胺可以被纳米线的某些晶面优先吸附而抑制相 应晶面的生长. 2012 年, Desai 等 ${ }^{[41]}$ 也利用氨水-聚乙烯 亚胺一六次甲基四胺一硝酸锌作为生长液, 在连续 $6 \mathrm{~h}$ 内 制备出了长度达到 $12 \mu \mathrm{m}$ 的 $\mathrm{ZnO}$ 纳米线阵列，纳米线表 面不仅没有纳米颗粒黏附，而且其根部保持独立而没有 融合在一起(图 8b). 因此, 协调生长液中氨水和聚乙烯 亚胺的浓度可以更好的控制纳米线的生长，从而制备出 高质量的超长 $\mathrm{ZnO}$ 纳米线阵列.

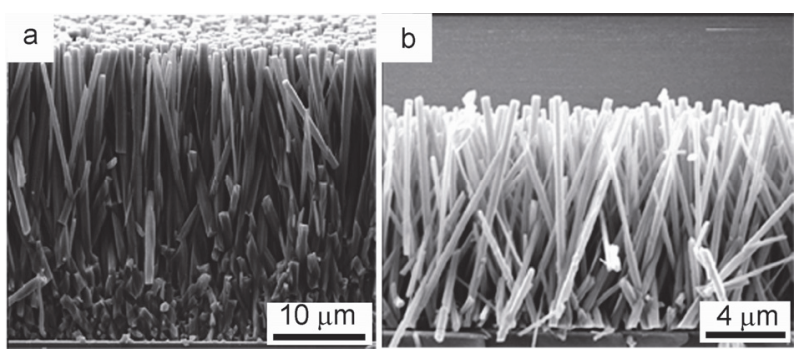

图 $8 \mathrm{ZnO}$ 纳米线阵列的 SEM 侧视图

Figure 8 Side-view SEM images of $\mathrm{ZnO}$ nanowire arrays (a) $30 \mu \mathrm{m}$ nanowire arrays ${ }^{[14]}$, (b) $12 \mu \mathrm{m}$ nanowire arrays ${ }^{[41]}$

综上所述，生长液中的氨水、六次甲基四胺、氯化 铵和聚乙烯亚胺等溶质对可控制备 $\mathrm{ZnO}$ 纳米线/棒阵列 具有重要作用，通过选取恰当的生长液组分，调节不同 溶质的浓度, 并选择合适的生长参数, 不仅可以快速有 效的制备出超长 $\mathrm{ZnO}$ 纳米线/棒阵列, 还可以解决纳米 线/棒阵列的根部融合问题.

\section{3 生长方法}

为了制备出长度较长的 $\mathrm{ZnO}$ 纳米线/棒阵列, 除了 可以选择合适的生长液, 还能在生长方法上进行改进. 由于生长方法的改进措施较多, 因而能够更好的实现 $\mathrm{ZnO}$ 纳米线/棒阵列的可控生长.

在制备 $\mathrm{ZnO}$ 纳米线/棒阵列的过程中, 生长液中的 溶质会被不断消耗而最终导致纳米线停止生长. 为了解 决这一问题, 2005 年 $\mathrm{Law}$ 等 ${ }^{[11]}$ 利用每隔 $2.5 \mathrm{~h}$ 更新一次 生长液的方法, 在 $50 \mathrm{~h}$ 内制备出了长度达到 $17 \mu \mathrm{m}$ 的 $\mathrm{ZnO}$ 纳米线阵列, 如图 9 所示. 由于更新生长液的方法 不仅可以有效地制备出长度较长的 $\mathrm{ZnO}$ 纳米线阵列, 而且实验操作相对简单，使得这种方法得到了较为广泛 的应用 ${ }^{[42 \sim 44]}$.

2009 年, Qiu 等 ${ }^{[34]}$ 对两步水热法的第二步进行了改 进，预先对生长液加热 $6 \mathrm{~h}$, 以抑制生长液的均相成核, 然后再将覆盖 $\mathrm{ZnO}$ 种子层的基底放入生长液中, 在 $10 \mathrm{~h}$ 内制备出了长度为 $10 \mu \mathrm{m}$, 长径比达到 150 的 $\mathrm{ZnO}$ 纳米 


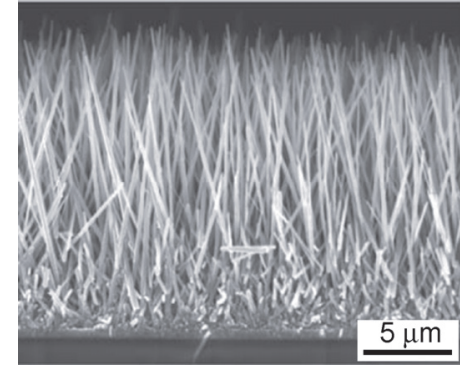

图 9 利用更新生长液而制备的 $\mathrm{ZnO}$ 纳米线阵列的 SEM 侧视图 ${ }^{[1]}$ Figure 9 Side-view SEM image of $\mathrm{ZnO}$ nanowire arrays prepared by refreshing growth solution ${ }^{[11]}$

线阵列(图 10), 并通过调节预加热时间对纳米线的直径 和长度进行了有效控制, 从而实现了 $\mathrm{ZnO}$ 纳米线阵列 的可控生长.

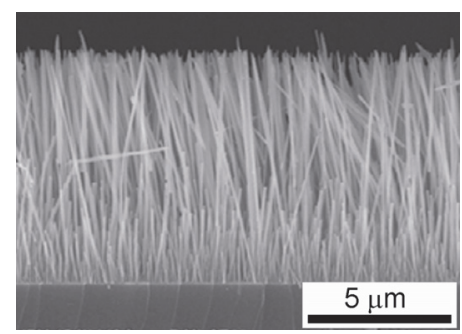

图 10 预先加热生长液 $6 \mathrm{~h}$ 制备的 $\mathrm{ZnO}$ 纳米线阵列的 $\mathrm{SEM}$ 侧视图 ${ }^{[34]}$ Figure 10 Side-view SEM image of $\mathrm{ZnO}$ nanowire arrays prepared by preheating growth solution for $6 \mathrm{~h}^{[34]}$

为了制备出更高长径比的超长 $\mathrm{ZnO}$ 纳米线阵列, 2010 年 Qiu 等 ${ }^{[6]}$ 除了预加热生长液, 还在生长液中添加 了聚乙烯亚胺, 制备出了长度为 $40 \mu \mathrm{m}$, 长径比高达 330 的 $\mathrm{ZnO}$ 纳米线阵列(图 11a). 研究表明, 种子层的反 复涂覆次数和预加热生长液的时间均会影响种子层表
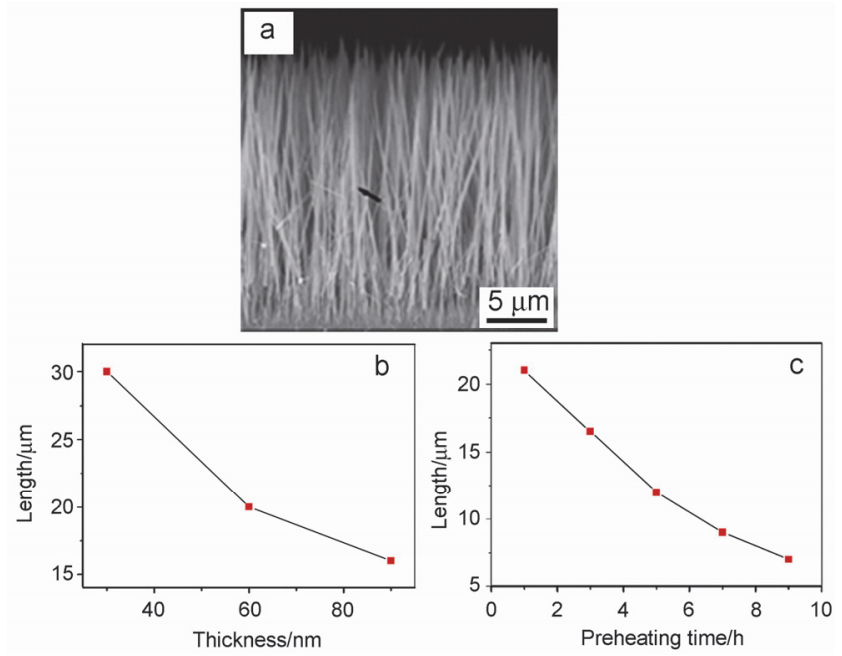

图 11 (a) $40 \mu \mathrm{m}$ 长的 $\mathrm{ZnO}$ 纳米线阵列的 SEM 侧视图、(b) $\mathrm{ZnO}$ 种子 层厚度与 $\mathrm{ZnO}$ 纳米线阵列长度之间的函数关系及(c)预加热生长液时 间与 $\mathrm{ZnO}$ 纳米线阵列长度之间的函数关系 ${ }^{[6]}$

Figure 11 (a) Side-view SEM image of a $40 \mu \mathrm{m} \mathrm{ZnO} \mathrm{nanowire} \mathrm{arrays,}$ (b) the functional relationship between length of $\mathrm{ZnO}$ nanowire arrays and thickness of $\mathrm{ZnO}$ seed layer and (c) the functional relationship between length of $\mathrm{ZnO}$ nanowire arrays and preheating time $\mathrm{e}^{[6]}$
面的粗糙度和结晶质量(图 11b), 进而影响 $\mathrm{ZnO}$ 纳米线 的长度和直径(图 11c).

在制备 $\mathrm{ZnO}$ 纳米线阵列的过程中, 粗而短的 $\mathrm{ZnO}$ 纳米线容易根部融合 (图 12a), 细而长的 $\mathrm{ZnO}$ 纳米线则 容易倾倒(图 12b), 为了解决这一问题, 2014 年 Deng 等 ${ }^{[45]}$ 首先利用氢氧化钠-硝酸锌作为生长液对覆盖 $\mathrm{ZnO}$ 种子层的基底预处理数分钟, 使生长初期的 $\mathrm{ZnO}$ 纳米 线较粗, 然后再利用氨水-聚乙烯亚胺一六次甲基四胺硝酸锌作为生长液进行正常的生长, 在 $10 \mathrm{~h}$ 内制备出了 长度为 $5 \mu \mathrm{m}$, 直径为 $150 \mathrm{~nm}$ 的 $\mathrm{ZnO}$ 纳米线阵列, 如图 $12 \mathrm{c}$ 所示. 该方法既避免了纳米线根部的融合, 还解决 了容易倾倒的问题. 同时，控制预处理时间还可以对 $\mathrm{ZnO}$ 纳米线的垂直度、直径以及密度进行有效的控制, 进而实现根部无融合的垂直 $\mathrm{ZnO}$ 纳米线阵列的可控制 备.

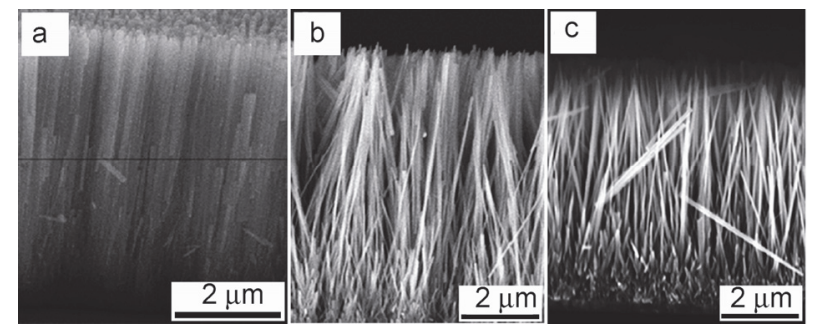

图 12 (a)根部融合的 $\mathrm{ZnO}$ 纳米线阵列、(b)倾倒的 $\mathrm{ZnO}$ 纳米线阵列及 (c)经过预处理后制备的 $\mathrm{ZnO}$ 纳米线阵列 ${ }^{[45]}$

Figure 12 (a) $\mathrm{ZnO}$ nanowire arrays with fusional roots, (b) sloped $\mathrm{ZnO}$ nanowire arrays and (c) $\mathrm{ZnO}$ nanowire arrays after pretreatment ${ }^{[45]}$

由于氨水具有抑制生长液均相成核的作用, 2012 年 Wang 等 ${ }^{[46]}$ 首先打开容器使氨气挥发而达到了降低氨水 浓度的目的，从而通过促进生长液表面的均相成核制备 出了 $\mathrm{ZnO}$ 种子层, 然后关闭容器以保持生长液中的氨 水浓度，使 $\mathrm{ZnO}$ 纳米线能够在均相成核的种子层上正 常生长, 制备过程如图 13a 所示. 该方法在 $2 \mathrm{~h}$ 内制备出 的 $\mathrm{ZnO}$ 纳米线阵列长度达到了 $10 \mu \mathrm{m}$, 直径为 $600 \sim 800$ $\mathrm{nm}$ (图 13b).
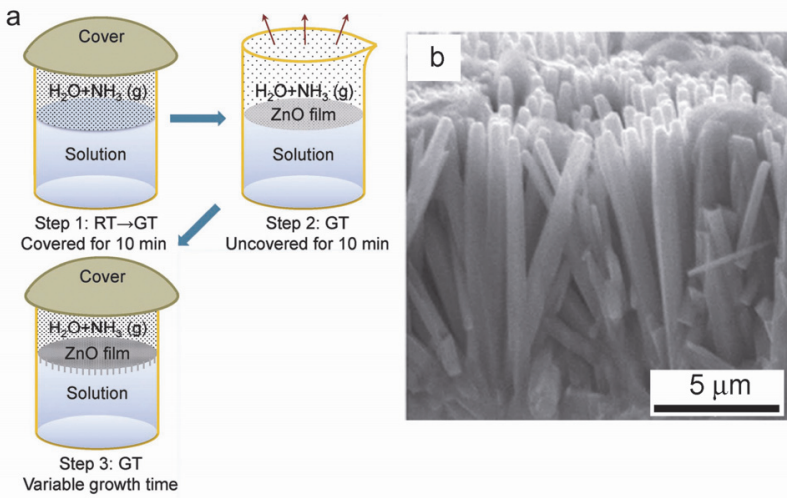

图 13 (a)水热法制备自支撑 $\mathrm{ZnO}$ 纳米线阵列的原理图及(b) $\mathrm{ZnO}$ 纳米 线阵列的 SEM 侧视图 ${ }^{[46]}$

Figure 13 (a) Schematic diagram of self-supporting $\mathrm{ZnO}$ nanowire arrays prepared by hydrothermal method and (b) side-view SEM image of $\mathrm{ZnO}$ nanowire arrays ${ }^{[46]}$ 
在制备超长 $\mathrm{ZnO}$ 纳米线阵列的过程中很容易出现 纳米线根部融合而导致表面积下降的问题，所以 2011 年 $\mathrm{Xu}$ 等 ${ }^{[24]}$ 将自组装技术和两步水热法相结合, 组装出 了多层 $\mathrm{ZnO}$ 纳米线阵列, 制备过程如图 14a 所示. 首先 在 $5 \mathrm{~h}$ 内制备出长度为 $10 \mu \mathrm{m}$, 直径为 $200 \mathrm{~nm}$ 的第一层 $\mathrm{ZnO}$ 纳米线阵列后, 利用自组装单层膜包裹纳米线的表 面而限制其径向生长, 然后再组装第二层纳米线阵列, 最终使得 $\mathrm{ZnO}$ 纳米线阵列的总长度达到了 $40 \mu \mathrm{m}$, 而且 制备出的纳米线阵列的根部没有出现融合(图 14b).

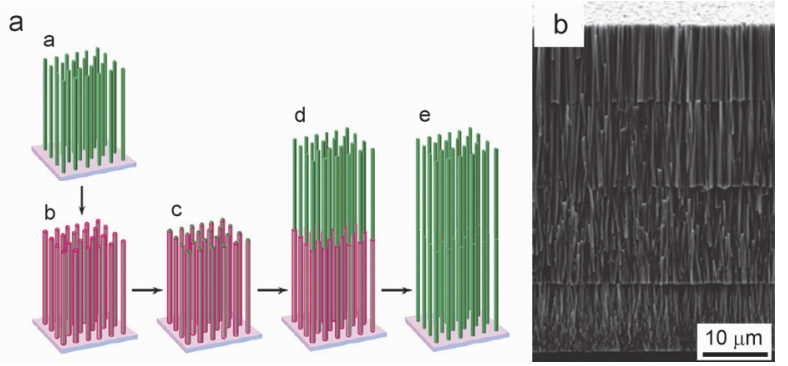

图 14 (a)组装两层 $\mathrm{ZnO}$ 纳米线阵列的原理图及 (b)四层 $\mathrm{ZnO}$ 纳米线阵 列的 SEM 侧视图 ${ }^{[24]}$

Figure 14 (a) Schematic diagram of a two-layer assembly of $\mathrm{ZnO}$ nanowire arrays and (b) side-view SEM image of a four-layer assembly of $\mathrm{ZnO}$ nanowire arrays ${ }^{[24]}$

生长液的温度和浓度对 $\mathrm{ZnO}$ 纳米线阵列的可控制 备具有重要作用, 所以 2012 年 $\mathrm{Xu}$ 等 ${ }^{[40]}$ 首先采用适当浓 度的种子液以控制纳米线阵列的密度, 然后将生长液第 一阶段的生长温度设定为 $88{ }^{\circ} \mathrm{C}$ 并维持 $10 \mathrm{~min}$, 以促进 大尺寸晶种生长为较粗的纳米线, 避免第二阶段低温生 长对其生长速率的影响; 而第二阶段的生长温度设定为 $85{ }^{\circ} \mathrm{C}$ 并生长 $12 \mathrm{~h}$, 以降低小尺寸晶种生长为短纳米线 的生长速率, 避免纳米线阵列中出现短纳米线而造成纳 米线阵列的根部融合; 同时还利用低浓度硝酸锌, 在质 量输运的作用下抑制短纳米线的生长和长纳米线的径 向生长. 最终制备出的 $\mathrm{ZnO}$ 纳米线阵列密度为 $8.3 \times 10^{8} \mathrm{~cm}^{-2}$, 长度达到 $22 \mu \mathrm{m}$, 长径比达到 80 , 而且 纳米线阵列的根部没有融合(图 15).

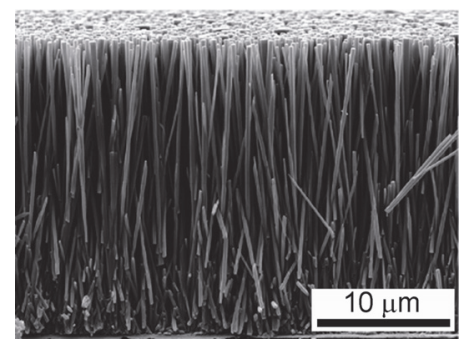

图 15 两步水热过程制备的 $\mathrm{ZnO}$ 纳米线阵列的 SEM 侧视图 ${ }^{[40]}$ Figure 15 Side-view SEM image of $\mathrm{ZnO}$ nanowire arrays prepared by two-step hydrothermal process ${ }^{[40]}$

由于制备 $\mathrm{ZnO}$ 纳米线阵列时会不断消耗生长液中 的溶质而阻碍纳米线的生长, 所以 2012 年 Chen 等 ${ }^{[47]}$ 利用连续流动注入的方法(图 16a) 在 $24 \mathrm{~h}$ 内制备了长度
为 $15 \mu \mathrm{m}$, 直径为 $100 \sim 150 \mathrm{~nm}$ 的 $\mathrm{ZnO}$ 纳米线阵列(图 $16 \mathrm{~b})$. 该方法不仅避免了不断更新生长液的问题，还减 少了纳米线的生长时间，而且制备出的 $\mathrm{ZnO}$ 纳米线阵 列的光学特性和结构质量都较好.
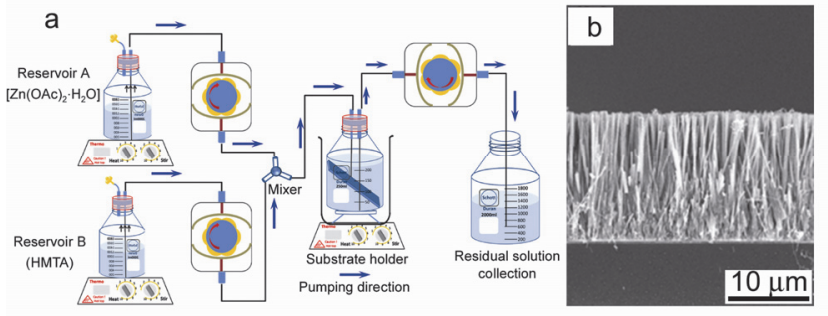

图 16 (a)连续流动注入装置的原理图及(b)锌前驱体浓度为 0.025 $\mathrm{mol} \cdot \mathrm{L}^{-1}$ 时制备出的 $\mathrm{ZnO}$ 纳米线阵列的 SEM 侧视图 ${ }^{[47]}$

Figure 16 (a) Schematic figure of the continuous flow injection apparatus and (b) side-view SEM image of $\mathrm{ZnO}$ nanowire arrays prepared when the zinc precursor concentration is $0.025 \mathrm{~mol} \cdot \mathrm{L}^{-1[47]}$

最近, 本课题组 ${ }^{[48]}$ 首先将 $0.0111 \mathrm{~g}$ 醋酸锌和 0.0060 $\mathrm{g}$ 的氢氧化钠溶解于 $12 \mathrm{~mL}$ 甲醇溶液中, 超声 $2 \mathrm{~h}$ 后形 成均匀的种子液; 然后在玻璃基底上旋涂种子液并加热 形成 $\mathrm{ZnO}$ 种子层，再利用微加工技术构建出了微流控 器件(图 17a); 最后在 $90{ }^{\circ} \mathrm{C}$ 条件下保持生长液的注入速 度为 $15 \mu \mathrm{L} / \mathrm{min}$, 在 $6 \mathrm{~h}$ 内制备出了长度达到 $11 \mu \mathrm{m}$ 的 $\mathrm{ZnO}$ 纳米线阵列(图 17b), 纳米线阵列的制备过程如图 $17 \mathrm{c}$. 该方法避免了更新生长液的复杂操作 ${ }^{[11]}$, 进一步 减少了 $\mathrm{ZnO}$ 纳米线阵列的制备时间，提高了纳米线的 生长效率 ${ }^{[37]}$.
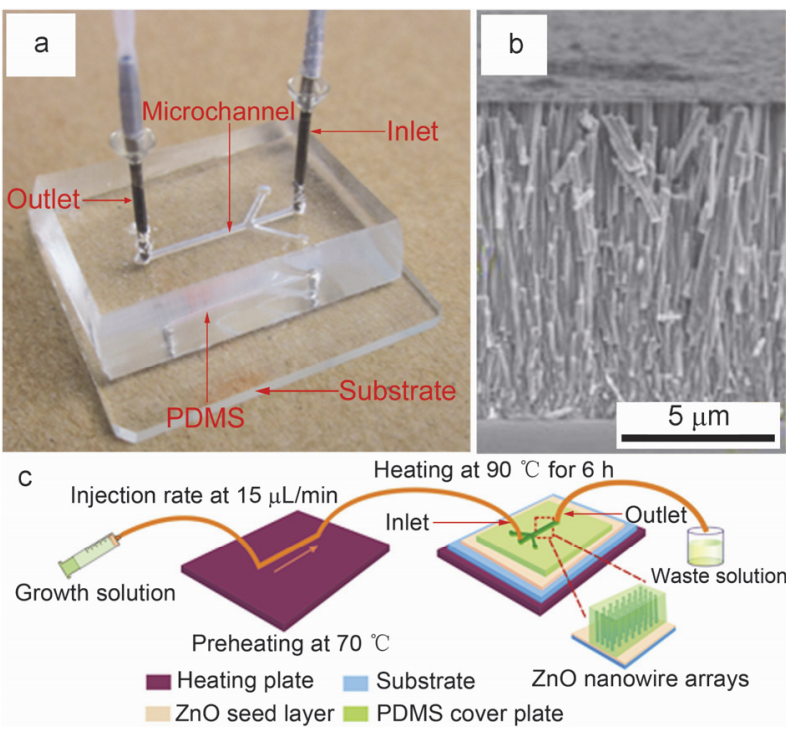

图 17 (a)制备 $\mathrm{ZnO}$ 纳米线阵列的微流控器件、(b)生长液流速为 15 $\mu \mathrm{L} / \mathrm{min}$ 时所制备 $\mathrm{ZnO}$ 纳米线阵列的 SEM 侧视图及(c)基于微流控技术 制备 $\mathrm{ZnO}$ 纳米线阵列的原理图 ${ }^{[48]}$

Figure 17 (a) Microfluidic device of preparing $\mathrm{ZnO}$ nanowire arrays, (b) side-view SEM image of $\mathrm{ZnO}$ nanowire arrays prepared when the velocity of growth solution was $15 \mu \mathrm{L} / \mathrm{min}$ and (c) schematic diagram of preparing $\mathrm{ZnO}$ nanowire arrays based on microfluidic technique ${ }^{[48]}$

综上所述, 选择合理的生长方法, 在短时间内不仅 
可以实现超长 $\mathrm{ZnO}$ 纳米线/棒阵列的制备, 还可以解决 纳米线/棒阵列的根部融合问题, 有助于制备出高比表 面积的超长 $\mathrm{ZnO}$ 纳米线/棒阵列, 提高各种光电器件的 性能.

\section{$3 \mathrm{ZnO}$ 纳米线/棒阵列的应用}

$\mathrm{ZnO}$ 纳米线/棒阵列具有比表面积大、电子输运快 和电子注入效率高等优点, 已经被广泛应用于染料敏化 太阳能电池、纳米发电机、气体传感器以及场发射器件 等领域.

\section{1 染料敏化太阳能电池}

传统的染料敏化太阳能电池主要以 $\mathrm{TiO}_{2}$ 纳米颗粒 薄膜作为光阳极, 但 $\mathrm{TiO}_{2}$ 纳米颗粒薄膜的颗粒晶界较 多, 且电子迁移率与 $\mathrm{ZnO}$ 相比较为缓慢, 导致大量光生 载流子复合, 阻碍了染料敏化太阳能电池性能的进一步 提高 ${ }^{[36,49]} . \mathrm{ZnO}$ 纳米线/棒阵列不仅具有更高的电子迁移 率 ${ }^{[37]}$, 而且还能为电子的快速传输提供一条直接的导 电通道 ${ }^{[50,51]}$, 确保光伏器件产生的载流子能够被快速收 集 ${ }^{[11]}$. 现阶段基于 $\mathrm{ZnO}$ 纳米线/棒阵列的染料敏化太阳 能电池已有所报道 ${ }^{[52,53]}$, 为了进一步提高这类电池的性 能, 可以通过增加 $\mathrm{ZnO}$ 纳米线/棒阵列的长度来实 现 $^{[36,41]}$.

2013 年 $\mathrm{Zhu}$ 等 ${ }^{[37]}$ 利用 $11 \mu \mathrm{m}$ 长的 $\mathrm{ZnO}$ 纳米线阵列 作为染料敏化太阳能电池的光阳极, 其功率转换效率为 $0.73 \% ; 2007$ 年 $\mathrm{Gao}$ 等 $^{[8]}$ 将 $\mathrm{ZnO}$ 纳米线阵列的长度提高 到 $14 \mu \mathrm{m}$ 后, 使染料敏化太阳能电池的功率转换效率达 到了 $1.70 \% ; 2010$ 年 $\mathrm{Xu}$ 等 ${ }^{[14]}$ 再次将纳米线长度增加至 $33 \mu \mathrm{m}$, 该器件的功率转换效率达到了 $2.10 \%$. 相应的功 率转换效率如表 1 所示.

表 1 基于不同长度 $\mathrm{ZnO}$ 纳米线阵列的染料敏化太阳能电池的功率转 换效率

Table 1 The power conversion efficiency of dye-sensitized solar cells based on $\mathrm{ZnO}$ nanowire arrays with different lengths

\begin{tabular}{cccccc}
\hline 阵列长度 $/ \mu \mathrm{m}$ & $P_{\mathrm{in}}{ }^{a} /\left(\mathrm{mW} \cdot \mathrm{cm}^{-2}\right)$ & $J_{\mathrm{sc}}{ }^{b} /\left(\mathrm{mA} \cdot \mathrm{cm}^{-2}\right)$ & $V_{\mathrm{oc}}{ }^{c} / \mathrm{V}$ & $\mathrm{FF}^{d}$ & $\eta / \%$ Ref. \\
\hline 11 & 100 & 2.901 & 0.641 & 0.393 & $0.73[37]$ \\
14 & 100 & 6.790 & 0.540 & 0.500 & $1.70[8]$ \\
33 & 100 & 9.300 & 0.672 & 0.341 & $2.10[14]$ \\
\hline
\end{tabular}

${ }^{a} P_{\text {in }}$ is the incident light power density; ${ }^{b} J_{\text {sc }}$ is the short circuit current; ${ }^{c} V_{\text {oc }}$ is the open circuit voltage; ${ }^{d} \mathrm{FF}$ is the fill factor.

因此，通过增加 $\mathrm{ZnO}$ 纳米线/棒阵列的长度，可以 增加染料的吸附量, 从而提高基于 $\mathrm{ZnO}$ 纳米线/棒阵列 的染料敏化太阳能电池的性能.

\section{2 纳米发电机}

基于 $\mathrm{ZnO}$ 纳米线阵列的半导体特性和压电特性, 2006 年 Wang 等 ${ }^{[54]}$ 发明了一种可以将机械能转换为电 能的纳米发电机, 由于这种压电器件在生物医学设备、 无线传感器以及便携式电子设备等方面具有潜在的应 用价值, 所以近年来基于 $\mathrm{ZnO}$ 纳米线阵列的纳米发电
机受到了较为广泛的关注 ${ }^{[55,56]}$.

2011 年 Wang 等 ${ }^{[57]}$ 利用不同长径比的 $\mathrm{ZnO}$ 纳米线 阵列制备了纳米发电机, 发现当 $\mathrm{ZnO}$ 纳米线阵列具有 适当的长径比和密度时, 可以使纳米发电机的性能达到 最佳. 2012 年 $\mathrm{Cho}$ 等 $^{[10]}$ 利用长度分别为 3.01, 6.09 和 $13.60 \mu \mathrm{m}$ 的 $\mathrm{ZnO}$ 纳米线阵列构建出了纳米发电机, 并且 发现当纳米线阵列的长度和长径比分别增加到 13.60 $\mu \mathrm{m}$ 和 97 时, 纳米发电机产生的电流密度可以达到 1.6 $\mu \mathrm{A} \cdot \mathrm{cm}^{-2}$, 如图 18 所示. 上述研究证明了增加 $\mathrm{ZnO}$ 纳 米线阵列的长度可以增加其长径比, 进而可以有效提高 纳米发电机的性能.

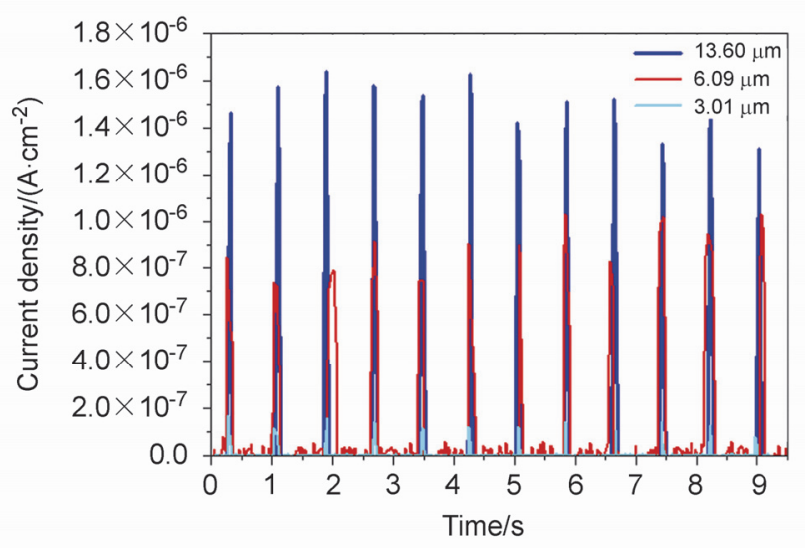

图 18 基于不同长度 $\mathrm{ZnO}$ 纳米线阵列的纳米发电机产生的电流密度 ${ }^{[10]}$ Figure 18 The current densities of nanogenerators based on $\mathrm{ZnO}$ nanowire arrays with different length ${ }^{[10]}$

因此，通过控制 $\mathrm{ZnO}$ 纳米线/棒阵列的生长，可以 获得长度更长的 $\mathrm{ZnO}$ 纳米线阵列, 这对进一步提高纳 米发电机的性能具有重要意义.

\section{3 气体传感器}

由于 $\mathrm{ZnO}$ 具有电子迁移率高、化学热稳定性好以 及对易燃易爆气体非常敏感等特性, 因而被广泛用作气 体传感器的敏感材料 ${ }^{[58,59]}$. 在 $\mathrm{ZnO}$ 材料的众多结构中, $\mathrm{ZnO}$ 纳米线/棒因其具有更高的电导率和更大的比表面 积, 近年来更成为了构建超高灵敏度气体传感器的理想 材料.

2010 年 Chang 等 ${ }^{[60]}$ 利用直径相同(50 80 nm), 长 度分别为 500 和 $1300 \mathrm{~nm}$ 的 $\mathrm{ZnO}$ 纳米棒阵列构建了二 氧化氮气体传感器, 结果表明纳米棒的长度越长, 气体 传感器的灵敏度越高(图 19a); 2009 年 Liu 等 ${ }^{[61]}$ 也利用直 径相同 $(30 \mathrm{~nm})$, 长度分别为 $1.78,1.43$ 和 $1.00 \mu \mathrm{m}$ 的 $\mathrm{ZnO}$ 纳米棒阵列构建了二氧化氮气体传感器, 结果表明气体 传感器的相对灵敏度随纳米棒阵列的长度增加而增加 (图 19b). 由分析可知, 气敏性能增强的主要原因是由于 纳米线/棒阵列的长度增加提高了材料的比表面积, 从 而为气体分子提供了更多的吸附位.

最近, 本课题组 ${ }^{[48]}$ 利用长度为 $11 \mu \mathrm{m}$ 的 $\mathrm{ZnO}$ 纳米阵 列作为气体传感器的敏感材料, 实现了对丙酮气体的气 

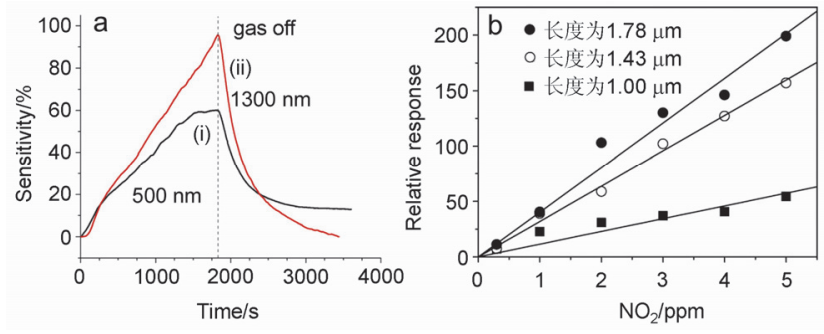

图 19 (a)基于不同长度 $\mathrm{ZnO}$ 纳米棒阵列气体传感器的响应 ${ }^{[60]}$ 及(b) 基于不同长度 $\mathrm{ZnO}$ 纳米棒阵列气体传感器的相对灵敏度 $\left.{ }^{[6]}\right]$

Figure 19 (a) Responses of gas sensors based on $\mathrm{ZnO}$ nanorod arrays with different length ${ }^{[60]}$ and (b) relative response of gas sensors based on $\mathrm{ZnO}$ nanorod arrays with different lengths ${ }^{[61]}$

敏性测试, 结果表明该气体传感器在 $475{ }^{\circ} \mathrm{C}$ 对 $200 \mathrm{ppm}$ 的丙酮气体具有较好的气敏性能, 其灵敏度达到了 8.26 , 相比传统水热法制备的纳米线阵列, 其气敏性能 有所提高. 所以增加 $\mathrm{ZnO}$ 纳米线阵列的长度, 可以进一 步提高该材料的气敏性能.

\section{4 场发射器件}

场发射器件广泛应用于电子显微镜、平板显示器和 $X$ 射线光源等领域. 自从发现碳纳米管 $(\mathrm{CNTs})$ 可以用于 高效的场发射器件之后, 具有良好机械稳定性和优良导 电性的一维 $\mathrm{ZnO}$ 纳米材料也备受关注. 研究发现, 场发 射器件的性能与其所使用 $\mathrm{ZnO}$ 纳米材料的密度和长径 比有很大的相关性, 所以对于 $\mathrm{ZnO}$ 的场发射特性研究 主要集中在如何通过对材料形貌的调控实现场增强因 子的提高 ${ }^{[62,63]}$.

2006 年 Wang 等 ${ }^{[64]}$ 制备了平均长度为 $3.3 \mathrm{~mm}$ 的 $\mathrm{ZnO}$ 纳米带阵列, 其电场仅为 $2.9 \mathrm{~V} / \mu \mathrm{m}$, 场增强因子达 到了 $1.4 \times 10^{4} ; 2007$ 年 Wang 等 ${ }^{[63]}$ 研究了不同密度的 $\mathrm{ZnO}$ 纳米线阵列的场发射特性, 发现当纳米线的密度为 $60 \sim 80 \mu \mathrm{m}^{-2}$, 长度为 $1 \mu \mathrm{m}$ 时, 器件能够获得较低的开 启电场及较高的发射电流, 如图 20 所示. 2008 年 Liu 等 ${ }^{[65]}$ 发现 $\mathrm{ZnO}$ 纳米线阵列的长度和长径比为 $1.03 \mu \mathrm{m}$ 和 26 , 密度 $1.0 \times 10^{9} \mathrm{~cm}^{-2}$ 时, 其场发射特性最好, 开启电 场仅为 $7.1 \mathrm{~V} / \mu \mathrm{m}$, 场增强因子达到了 862. 2013 年 Hsiao 等 ${ }^{[66]}$ 利用两步水热法在玻璃基底上制备出了长度和直 径为 $2.4 \mu \mathrm{m}$ 和 $120 \mathrm{~nm}$ 的镓掺杂 $\mathrm{ZnO}$ 纳米棒阵列, 并且 其开启电场仅为 $3.63 \mathrm{~V} / \mu \mathrm{m}$, 场增强因子达到了 9058 . 上述结果表明, 掺杂可以进一步提高 $\mathrm{ZnO}$ 纳米棒的场 发射性能.

因此, 通过控制 $\mathrm{ZnO}$ 纳米材料的生长条件, 可以使 纳米材料具有更加合适的密度和长径比, 从而使其性能 得到进一步提高.

\section{4 总结和展望}

综上所述, 两步水热法中的种子层、生长液组分以 及生长方法对平面基底上制备垂直均匀的超长 $\mathrm{ZnO}$ 纳

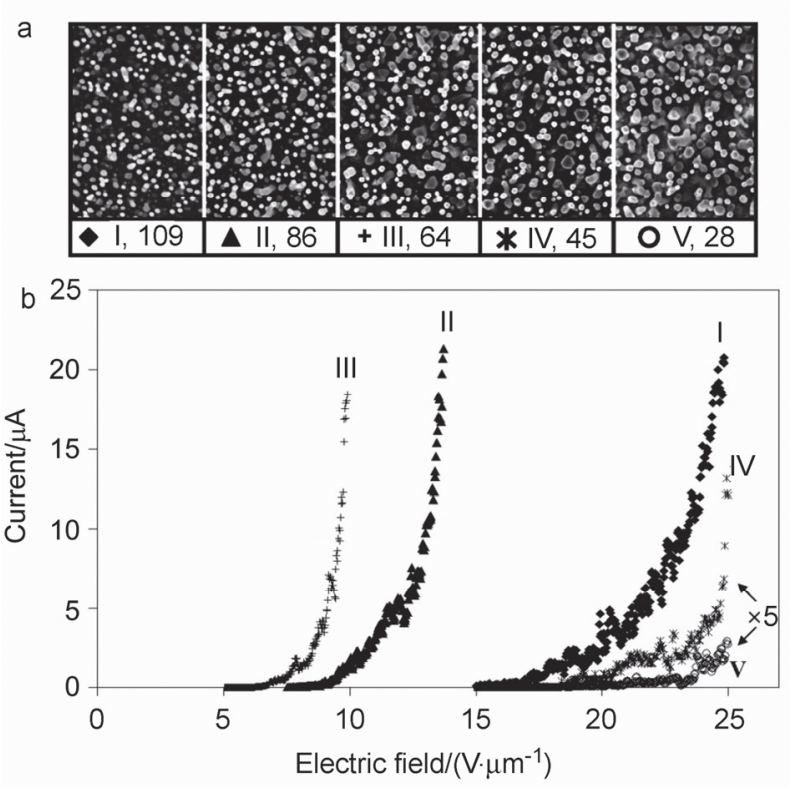

图 20 (a)不同密度的 $\mathrm{ZnO}$ 纳米线阵列的 SEM 俯视图及(b)相对应的 场发射 $I-V$ 曲线(其中曲线 IV 和 $\mathrm{V}$ 被放大了五倍) ${ }^{[63]}$

Figure 20 (a) Top-view SEM images of $\mathrm{ZnO}$ nanowire arrays with different densities and (b) corresponding field-emission $I-V$ characteristics curves (curves IV and V were magnified five times) ${ }^{[63]}$

米线阵列具有重要作用, 而且高质量的 $\mathrm{ZnO}$ 纳米线阵 列还可以进一步提高光电器件的性能, 因此未来的研究 重点仍将着眼于制备方法的进一步优化. 目前利用两步 水热法在短时间内制备出垂直均匀的超长 $\mathrm{ZnO}$ 纳米线/ 棒阵列仍然较为困难, 而且制备出的纳米线/棒阵列还 存在根部融合而导致比表面积降低的问题, 所以可以对 本文列举的方法进行综合利用而提出新的制备方法, 以 快速制备出根部没有融合的垂直均匀的超长 $\mathrm{ZnO}$ 纳米 线/棒阵列. 此外, 为了进一步提高气体传感器的性能, 还可以对基于超长 $\mathrm{ZnO}$ 纳米线/棒阵列的气体传感器进 行结构优化, 构建出新型的气体传感器.

总之，如果能够找到更好的制备方法来快速制备垂 直均匀的超长 $\mathrm{ZnO}$ 纳米线/棒阵列, 并解决根部融合问 题, 不仅可以有效提高染料敏化太阳能电池和气体传感 器等器件的性能, 还可以进一步拓宽其应用领域, 实现 新的应用价值而造福于人类.

\section{作者简介}

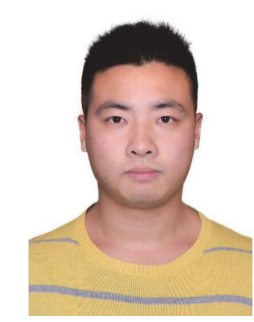

郝锐, 男, 1991 年出生, 本科毕业于太原理工大学物理与 光电工程学院, 自 2013 年起在太原理工大学微纳系统研究中 
心从事研究工作, 主要研究方向为微流控系统的设计及氧化 锌纳米线阵列的制备, 现为中国科学院电子学研究所硕士研 究生.

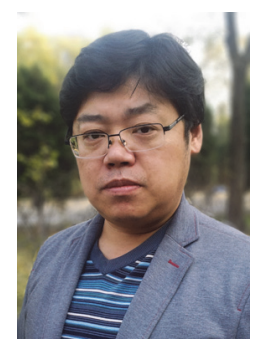

邓霄, 男, 1980 年出生, 太原理工大学副教授. 2014 年获 太原理工大学电路与系统博士学位, 导师为张文栋教授. 主 要从事功能纳米材料的合成、微纳传感器及测试系统等方面 的研究.

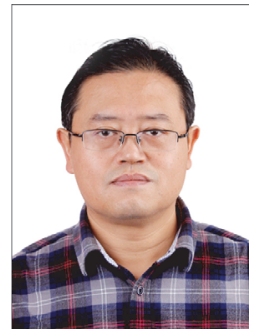

杨毅彪, 男, 1967 年出生, 教授. 2009 年获太原理工大学 材料加工工程专业博士学位. 主要从事光子晶体、太阳能电池 的设计与制备等方面的研究工作.

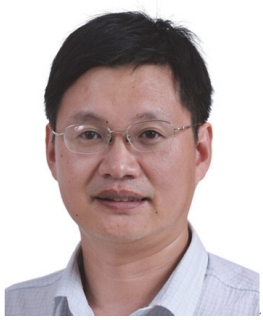

陈德勇, 男, 1967 年出生, 研究员. 2002 年获中国科学院 电子学研究所物理电子学博士学位. 主要从事 MEMS 微传感 器与微系统、微流控芯片系统等方面的研究工作.

\section{References}

[1] Zhou, X.-Y. Ph.D. Dissertation, China University of Petroleum (East China), Qingdao, 2011. (周小岩, 博士论文, 中国石油大学 (华东), 青岛, 2011.)

[2] Xiong, J.-F.; Xiao, P.; Wu, Q.; Wang, X.-Z.; Hu, Z. Acta Chim. Sinica 2014, 72, 433. (熊静芳, 肖佩, 吴强, 王喜章, 胡征, 化学 学报, 2014, 72, 433.)

[3] Zhang, Y. One-dimensional ZnO Nanomaterials, Science Press, Beijing, 2010, pp. 1 3. (张跃, 一维氧化锌纳米材料, 科学出版 社, 北京, 2010 , pp. 1 3.)

[4] He, Y.; Wang, J.-A.; Sang, W.-B.; Wu, R.-F.; Yan, L.-L.; Fang, Y.-Y. Acta Chim. Sinica 2005, 63, 1037. (贺英, 王均安, 桑文斌, 吴若峰, 颜莉莉, 方云英, 化学学报, 2005, 63, 1037.)

[5] Lee, Y. M.; Yang, H. W. J. Solid State Chem. 2011, 184, 615.

[6] Qiu, J.-J.; Li, X.-M.; Zhuge, F. W.; Gan, X.-Y.; Gao, X.-D.; He, W.-Z.; Park, S. J.; Kim, H. K.; Hwang, Y. H. Nanotechnology 2010 , 21, 195602.

[7] Wang, J.-X.; Sun, X.-W.; Yang, Y.; Huang, H.; Lee, Y. C.; Tan, O.-K.; Vayssieres, L. Nanotechnology 2006, 17, 4995.

[8] Gao, Y.-F.; Nagai, M.; Chang, T. C.; Shyue, J. J. Cryst. Growth Des. 2007, 7, 2467.
[9] Lupan, O.; Pauporté, T.; Viana, B. Adv. Mater. 2010, 22, 3298.

[10] Cho, J. W.; Lee, C. S.; Lee, K. I.; Kim, S. M.; Kim, S. H.; Kim, Y. K. Appl. Phys. Lett. 2012, 101, 083905.

[11] Law, M.; Greene, L. E.; Johnson, J. C.; Saykally, R.; Yang, P.-D. Nat. Mater. 2005, 4, 455.

[12] Chen, C. H.; Chang, S. J.; Chang, S. P.; Li, M. J.; Chen, I.; Hsueh, T. J.; Hsu, C. L. Chem. Phys. Lett. 2009, 476, 69.

[13] Hsueh, T. J.; Chen, Y. W.; Chang, S. J.; Wang, S. F.; Hsu, C. L.; Lin, Y. R.; Lin, T. S.; Chen, I. Sens. Actuators, B 2007, 125, 498.

[14] Xu, C.-K.; Shin, P.; Cao, L.-L.; Gao, D. J. Phys. Chem. C 2010, $114,125$.

[15] Greene, L. E.; Law, M.; Tan, D. H.; Montano, M.; Goldberger, J.; Somorjai, G.; Yang, P.-D. Nano Lett. 2005, 5, 1231.

[16] Wang, L.-S.; Zhang, X.-Z.; Zhao, S.-Q.; Zhou, G.-Y.; Zhou, Y.-L.; Qi, J.-J. Appl. Phys. Lett. 2005, 86, 024108.

[17] Tien, L. C.; Pearton, S. J.; Norton, D. P.; Ren, F. J. Mater. Sci. 2008, 43, 6925 .

[18] Guo, M.; Diao, P.; Cai, S.-M. Acta Chim. Sinica 2003, 61, 1165. (郭 敏, フ鹏, 蔡生民, 化学学报, 2003, 61, 1165.)

[19] Elias, J.; Tena Zaera, R.; Lévy Clément, C. J. Electroanal. Chem. 2008, 621, 171.

[20] Fan, H. J.; Lee, W.; Scholz, R.; Dadgar, A.; Krost, A.; Nielsch, K.; Zacharias, M. Nanotechnology 2005, 16, 913.

[21] Zhang, Y. One-dimensional ZnO Nanomaterials, Science Press, Beijing, 2010, pp. 104 106. (张跃, 一维氧化锌纳米材料, 科学 出版社, 北京, 2010, pp. 104 106.)

[22] Vayssieres, L. Adv. Mater. 2003, 15, 464.

[23] Vayssieres, L.; Keis, K.; Lindquist, S. E.; Hagfeldt, A. J. Phys. Chem. B 2001, 105, 3350.

[24] Xu, C.-K.; Wu, J.-M.; Desai, U. V.; Gao, D. J. Am. Chem. Soc. 2011, 133, 8122 .

[25] Liou, S. C.; Hsiao, C. S.; Chen, S. Y. J. Cryst. Growth 2005, 274, 438.

[26] Tao, Y.-L.; Fu, M.; Zhao, A.-L.; He, D.-W.; Wang, Y.-S. J. Alloys Compd. 2010, 489, 99.

[27] Sun, Y.; George Ndifor Angwafor, N.; Jason Riley, D.; Ashfold, M. N. R. Chem. Phys. Lett. 2006, 431, 352.

[28] Zheng, J.-H.; Zhang, X.-K.; Lu, H.-F. Acta Chim. Sinica 2011, 69, 2434. (郑建华，张晓凯，卢慧粉，化学学报, 2011, 69, 2434.)

[29] Huang, Q.-L.; Fang, L.; Chen, X.; Saleem, M. J. Alloys Compd. 2011, 509, 9456.

[30] Tan, Y.-W.; Xue, X.-Y.; Peng, Q.; Zhao, H.; Wang, T.-H.; Li, Y.-D. Nano Lett. 2007, 7, 3723.

[31] Yu, Y.-X.; Hao, W.-C.; Du, Y.; Wang, C.-Z.; Wang, T.-M. J. Nanosci. Nanotechnol. 2009, 9, 909.

[32] Xu, S.; Wang, Z.-L. Nano Res. 2011, 4, 1013.

[33] Greene, L. E.; Yuhas, B. D.; Law, M.; Zitoun, D.; Yang, P.-D. Inorg. Chem. 2006, 45, 7535.

[34] Qiu, J.-J.; Li, X.-M.; He, W.-Z.; Park, S. J.; Kim, H. K.; Hwang, Y. H.; Lee, J. H.; Kim, Y. D. Nanotechnology 2009, 20, 155603.

[35] Sugunan, A.; Warad, H. C.; Boman, M.; Dutta, J. J. Sol-Gel Sci. Technol. 2006, 39, 49.

[36] Chen, L.-L.; Li, X.-D.; Qu, L.-L.; Gao, C.-T.; Wang, Y.-Q.; Teng, F.; Zhang, Z.-X.; Pan, X.-J.; Xie, E.-Q. J. Alloys Compd. 2014, 586, 766.

[37] Zhu, S.-B.; Chen, X.-N.; Zuo, F.-B.; Jiang, M.; Zhou, Z.-W.; Hui, D. J. Solid State Chem. 2013, 197, 69.

[38] Zhou, Y.; Wu, W.-B.; Hu, G.-D.; Wu, H.-T.; Cui, S.-G. Mater. Res. Bull. 2008, 43, 2113.

[39] Yin, X.-T.; Que, W.-X.; Fei, D.; Xie, H.-X.; He, Z.-L.; Wang, G.-F. Electrochim. Acta 2013, 89, 561.

[40] Xu, C.-K.; Gao, D. J. Phys. Chem. C 2012, 116, 7236.

[41] Desai, U. V.; Xu, C.-K.; Wu, J.-M.; Gao, D. Nanotechnology 2012, 23, 205401.

[42] Xu, C.; Wang, X.-D.; Wang, Z.-L. J. Am. Chem. Soc. 2009, 131, 5866.

[43] Hsu, Y. F.; Xi, Y. Y.; Djurišić, A. B.; Chan, W. K. Appl. Phys. Lett. 2008, 92, 133507.

[44] Gao, H.-M.; Fang, G.-J.; Wang, M.-J.; Liu, N.-S.; Yuan, L.-Y.; Li, C.; Ai, L.; Zhang, J.; Zhou, C.-H.; Wu, S.-J. Mater. Res. Bull. 2008, 43,3345 .

[45] Deng, J.-P.; Wang, M.-Q.; Song, X.-H.; Liu, J. J. Alloys Compd. 2014, $588,399$.

[46] Wang, L. S.; Tsan, D.; Stoeber, B.; Walus, K. Adv. Mater. 2012, 24, 3999.

[47] Chen, L. Y.; Yin, Y. T. Cryst. Growth Des. 2012, 12, 1055. 
[48] Hu, J.; Deng, X.; Sang, S.-B.; Li, P.-W.; Li, G.; Zhang, W.-D. Acta Phys. Sin. 2014, 63, 207102-1. (胡杰, 邓霄, 桑胜波, 李朋伟, 李 刚, 张文栋, 物理学报, 2014, 63, 207102-1.)

[49] Gao, C.-T.; Li, X.-D.; Wang, Y.-Q.; Chen, L.-L.; Pan, X.-J.; Zhang, Z.-X.; Xie, E.-Q. J. Power Sources 2013, 239, 458.

[50] Hochbaum, A. I.; Yang, P.-D. Chem. Rev. 2009, 110, 527.

[51] Pradhan, B.; Batabyal, S. K.; Pal, A. J. Sol. Energy Mater. Sol. Cells 2007, 91, 769 .

[52] Baxter, J. B.; Aydil, E. S. Appl. Phys. Lett. 2005, 86, 053114.

[53] Qin, Z.; Liao, Q.-L.; Huang, Y.-H.; Tang, L.-D.; Zhang, X.-H.; Zhang, Y. Mater. Chem. Phys. 2010, 123, 811.

[54] Wang, Z.-L.; Song, J.-H. Science 2006, 312, 242.

[55] Zhu, G.; Yang, R.-S.; Wang, S.-H.; Wang, Z.-L. Nano Lett. 2010, $10,3151$.

[56] Lu, M. P.; Song, J.-H.; Lu, M. Y.; Chen, M. T.; Gao, Y.-F.; Chen, L. J.; Wang, Z.-L. Nano Lett. 2009, 9, 1223.

[57] Riaz, M.; Song, J.-h.; Nur, O.; Wang, Z.-L.; Willander, M. $A d v$. Funct. Mater. 2011, 21, 628.

[58] Liao, L.; Lu, H.-B.; Li, J.-C.; He, H.; Wang, D.-F.; Fu, D.-J.; Liu, C.; Zhang, W.-F. J. Phys. Chem. C 2007, 111, 1900.
[59] Chang, C. M.; Hon, M. H.; Leu, I. C. Sens. Actuators, B 2010, 151, 15.

[60] Chang, C. J.; Hung, S. T.; Lin, C. K.; Chen, C. Y.; Kuo, E. H. Thin Solid Films 2010, 519, 1693.

[61] Liu, F. T.; Gao, S. F.; Pei, S. K.; Tseng, S. C.; Liu, C. H. J. J. Taiwan Inst. Chem. Eng. 2009, 40, 528.

[62] Liu, J.-P.; Xu, C.-X.; Zhu, G.-P.; Li, X.; Cui, Y.-P.; Yang, Y.; Sun, X.-W. J. Phys. D: Appl. Phys. 2007, 40, 1906.

[63] Wang, X.-D.; Zhou, J.; Lao, C.-S.; Song, J.-H.; Xu, N.-S.; Wang, Z.-L. Adv. Mater. 2007, 19, 1627.

[64] Wang, W.-Z.; Zeng, B.-Q.; Yang, J.; Poudel, B.; Huang, J.; Naughton, M. J.; Ren, Z. Adv. Mater. 2006, 18, 3275.

[65] Liu, J.; She, J.-C.; Deng, S.-Z.; Chen, J.; Xu, N. S. J. Phys. Chem. C 2008, 112, 11685.

[66] Hsiao, C. H.; Huang, C. S.; Young, S. J.; Chang, S. J.; Guo, J. J.; Liu, C. W.; Yang, T. Y. IEEE Trans. Electron Devices 2013, 60, 1905.

(Zhao, C.) 